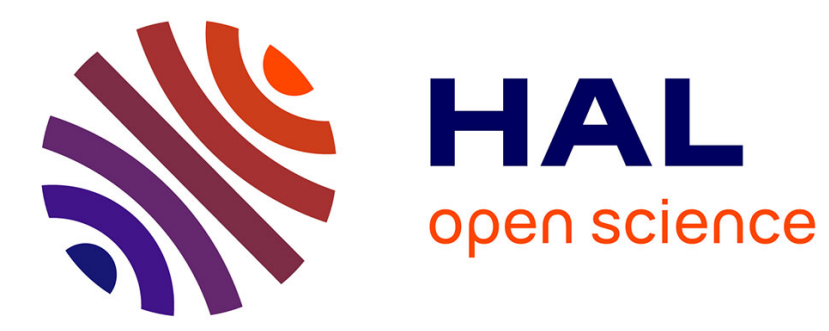

\title{
Effect of Illumination Intensity on LED Based Visible Light Communication System
}

Dayu Shi, Jiacheng Li, Yourong Liu, Lina Shi, Yanqi Huang, Zhan Wang, Xun Zhang, Andrei Vladimirescu

\section{- To cite this version:}

Dayu Shi, Jiacheng Li, Yourong Liu, Lina Shi, Yanqi Huang, et al.. Effect of Illumination Intensity on LED Based Visible Light Communication System. 15th Symposium on Broadband Multimedia Systems and Broadcasting 2020, Oct 2020, Paris, France. hal-02865817

\section{HAL Id: hal-02865817 https://hal.science/hal-02865817}

Submitted on 12 Jun 2020

HAL is a multi-disciplinary open access archive for the deposit and dissemination of scientific research documents, whether they are published or not. The documents may come from teaching and research institutions in France or abroad, or from public or private research centers.
L'archive ouverte pluridisciplinaire HAL, est destinée au dépôt et à la diffusion de documents scientifiques de niveau recherche, publiés ou non, émanant des établissements d'enseignement et de recherche français ou étrangers, des laboratoires publics ou privés. 


\title{
Effect of Illumination Intensity on LED Based Visible Light Communication System
}

\author{
Dayu Shi*, Jiacheng Li, Yourong Liu, Lina Shi, Yanqi Huang, Zhan Wang, Xun Zhang, Andrei Vladimirescu \\ Laboratory LISITE, Institut superieur d'electronique de Paris, France \\ *Contact: dayu.shi@isep.fr
}

\begin{abstract}
This paper addresses the issue that lighting requirements impose on Visible Light Communication (VLC) systems in terms of the power transfer function and the Signal Noise Ratio (SNR). The effect of illumination intensity on Visible Light Communication has been investigated theoretically and experimentally based on our white LED-based system model. The communication quality declined by an increase of the illumination intensity of LED through the simulation and the experiment. When the illuminance of LED increased from $400 \mathrm{~lx}$ to $7000 \mathrm{~lx}$, the system SNR decreased significantly from $19.4 \mathrm{~dB}$ to $6.3 \mathrm{~dB}$. Meanwhile, the average power loss increases from $1.9 \mathrm{~dB}$ to 11.1 dB respectively.
\end{abstract}

Index Terms-Visible Light Communication (VLC), LED, Illumination Intensity, VLC system model

\section{INTRODUCTION}

New high-data-rate multimedia services and applications are evolving continuously and exponentially increasing the demand for wireless capacity of fifth-generation $(5 \mathrm{G})$ and beyond [1]. It is expected that $5 \mathrm{G}$ communication will comprise ultradense heterogeneous networks for which mobile data volume per area will be 1000 times and the number of connected wireless devices will be 100 times higher compared to those in existing wireless networks [2]. Therefore, building up high data rates, low power consumption, and negligible end-toend delays wireless communication links with the demand of ever increasing data traffic is challenging. As the radio frequency (RF) spectrum gets congested [3]. The idea of the optical wireless communication (OWC) has been presented as a promising solution for upcoming high-density and high capacity 5G wireless networks [1]. The OWC provides the nomadic access in hundreds of terahertz $(\mathrm{THz})$ of unlicensed optical spectrum, immunity to electromagnetic interference, safety and security, simple implementation and deployment of systems [4].

Meanwhile, due to the large-scale deployment of the white light-emitting-diode (LED) as the next-generation green lighting, the visible light communication (VLC), a subset of OWC, has emerged as a promising technology. The VLC technology is motivated by several benefits including, worldwide available and unlicensed bandwidth, non-interference with radio frequency bands, no electromagnetic radiation, and high data rate (up to $100 \mathrm{Gbps}$ ) [5]. These exciting assets generate considerable research and industrial interests for indoor VLC, especially with the approval of the IEEE 802.15.7 standard [9], hospital scenario [10] and future 5G solution [11].
Due to the dual functionality of VLC system which are illumination and data transmission. Beside the optical detector, VLC transmission must be cognizant of a second type of signal receiver - the human eye. Most of the researches primarily focus on achieving increasingly high data rates [5]. However, most of these demonstrations are implemented in laboratory environment which do not consider the lighting quality issues. Respecting of the European Lighting Standard EN12464-1 [14], it requires an illuminance of $500 \mathrm{~lx}$ for working area, a minimum illuminance of $50 \mathrm{~lx}$ for indirect light in the ceiling and vertical light on walls. Hence, dealing with perceived illumination constraints while achieving high data rates is a clear objective to make ubiquitous VLC systems visible [15].

Additionally, some researches have successfully achieved analyzing the effect of illumination on VLC system [4] [15]. However, most of these existed analysis pay more attention to the optical field distribution and light propagation, which might ignore the role of the transmitter device. Based on our proposal, it is obvious to perceive that the communication quality has been extremely affected by the illumination intensity of the transmitter device.

In this context, this paper propose a system model to theoretically analyze and experimentally demonstrate the effect of the illumination intensity on LED based VLC system. The main contributions of this paper are summarized as follows:

- This paper propose a VLC system model considering the LED device which can characterize the illumination intensity and the communication performance.

- Based on this system model, the quantitative relationship between the illumination intensity and the VLC system performance has been illustrated.

- The experimental results based simulation demonstrates the performance of VLC system under the influence of different illumination intensity, including the Signal Noise Ratio (SNR) and power transfer function.

In the remainder of this paper is organized as following: the section II proposed the system model in detail. The section III gives the experiment and results. The conclusion and discussion is presented in the section IV.

\section{SySTEM MOdEL}

Our proposal is applied on a white LED based VLC system scenario shown in Fig.1. Where illustration and communication link are both provided by LEDs. The modulated signal transmitted by a LED and received by a photodiode through an 


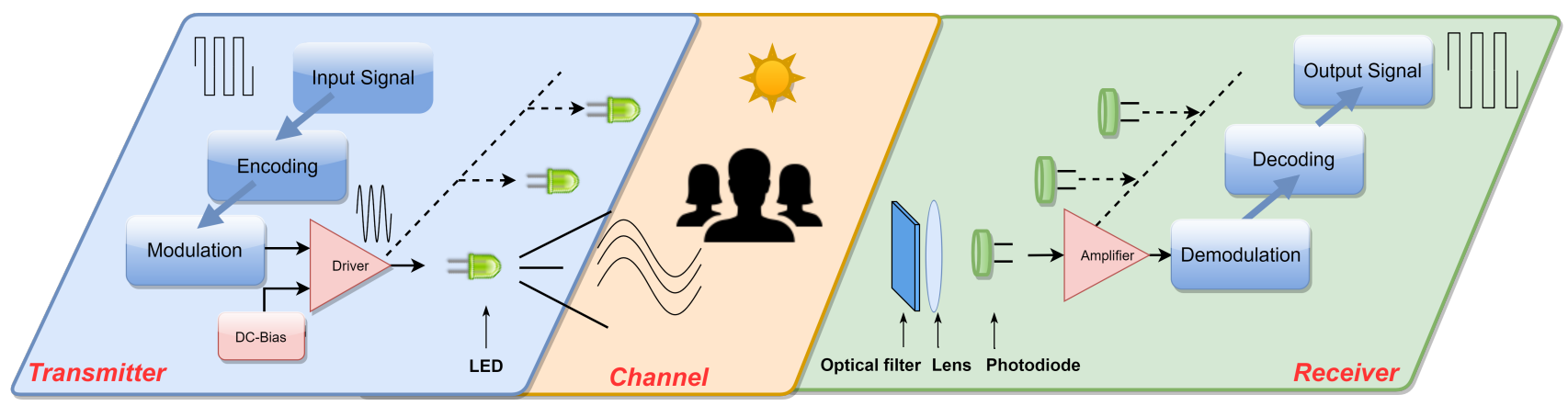

Fig. 1: A Typical VLC System Link

optical channel, an optical filter and a lens. After demodulation and decoding, users can received perceived signal. In this paper, we choose a typical InGaN based white LED as the transmitter of VLC system.

\section{A. Illumination Intensity and Power Transfer Function of LED}

The characteristic of a typical white LED is mainly caused by the intrinsic $p-n$ junction and the parasitic parameters which are due to the encapsulation and fabrication of LEDs. A small signal equivalent circuit of LED device is depicted in Fig.2.

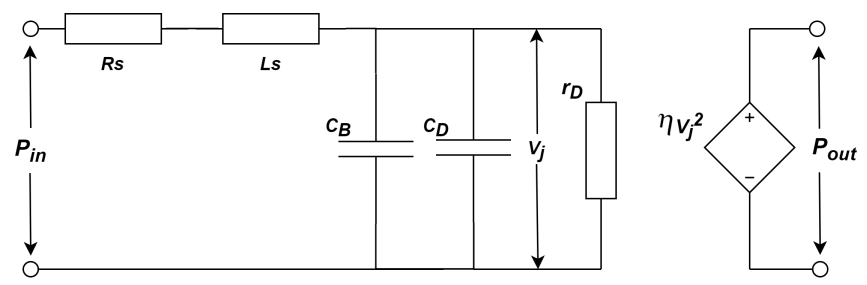

Fig. 2: A small signal equivalent circuit of LED device

where, $R_{s}$ and $L_{s}$ are the parasitic resistance and inductance. The $C_{B}$ and $C_{D}$ are the barrier capacitance and the diffusion capacitance. The $V_{j}$ is the junction voltage and the $r_{D}$ is the small signal resistance of p-n junction. The $\eta$ is the electro-optical conversion efficient.

Based on this equivalent circuit, the illumination intensity of the LED device $P_{\text {optical }}$ can be represented by the total output optical power which is consisted of the signal power $P_{\text {out }}(j \omega)$ and the DC power $P_{Q}$ shown in equation (1)

$$
\begin{gathered}
P_{\text {optical }}=P_{\text {out }}(j \omega)+P_{Q} \\
P_{\text {out }}(j \omega)=P_{\text {in }}(j \omega) * H_{\text {led }}(j \omega) \\
H_{\text {led }}(j \omega)=\frac{\eta * r_{D} Z_{\text {in }}}{\left(\left(j \omega r_{D} C+1\right)\left(R_{s}+Z_{\text {in }}+j \omega L\right)+r_{D}\right)^{2}} \\
I_{Q}=I_{s}\left(e^{\left(V_{Q}-I_{Q} R_{s}\right) / n V_{T}}-1\right) \\
r_{D}=\frac{d V_{Q}}{d I_{Q}}=\frac{n V_{T}}{I_{Q}}+R_{s}
\end{gathered}
$$

The power of output optical signal and the power transfer function can be represented by equation(2) (3). The $P_{i n}$ is the power of input electrical signal and the $P_{\text {out }}$ is the power of output optical signal. The $Z_{i n}$ is the input impedance and $C$ represent the sum of the barrier capacitance and the diffusion capacitance. The $\omega$ is the angular frequency of the signal.

By respecting the Shockley function, the I-V relationship at quiescent point can be represented by equation (4) [16]. When the quiescent junction current $I_{Q}$ is much larger than saturation current $I_{s}$, the small signal resistance of p-n junction can be illustrated as equation (5).

\section{B. Power Transfer Function of VLC System Link}

Once the LED device has been characterized, the propagation and detection of the light are demanded. In this paper, we assume that the indoor VLC system channel is the line-of-sight (LOS) channel and the light propagation is the Lambertian directional.

Under this assumption, the power transfer function of the optical channel can be represented by the equation(6) [17].

where $m$ is the Lambert's mode number expressing directivity of the source beam, $\phi$ is the angle of radiation incident maximum radiated power, $D$ is the distance between transmitter and receiver, Adet is effective the area of photodiode in receiver.

Thus, the whole VLC system link power transfer function which consist of transmitter, channel and receiver can be illustrated by the equation (7).

$$
\begin{gathered}
H_{c}=\frac{(m+1) * \operatorname{Adet} * \cos \phi^{m+1}}{2 \pi D^{2}} \\
H_{v l c}=H_{l e d} * H_{c} * H_{r}
\end{gathered}
$$

Where, the transfer function of receiver $H_{r}$ which consists of optical filter, lens, and photodiode can be supplied by the manufacture.

\section{Effect of illumination intensity on VLC system}

This subsection investigated the impact of illumination intensity on VLC system by using the above mentioned power transfer function of VLC system link. According to equations from (2) to (7), the received signal power $P_{r}$ and 
the illumination intensity of LED $P_{\text {optical }}$ can be represented by equation (8) (9).

$$
\begin{gathered}
P_{r}(j \omega)=P_{i n}(j \omega) * H_{v l c}\left(j \omega, I_{Q}, D, \phi\right) \\
P_{\text {optical }}=P_{i n}(j \omega) * H_{l e d}\left(j \omega, I_{Q}\right)+I_{Q} V_{Q} \eta
\end{gathered}
$$

We set the fixed location, signal frequency, and power of input signal to do the simulation to observe the relationship between the illumination intensity $P_{\text {optical }}$ and the received signal power $P_{r}$. The simulation result has been depicted in the Fig.3.

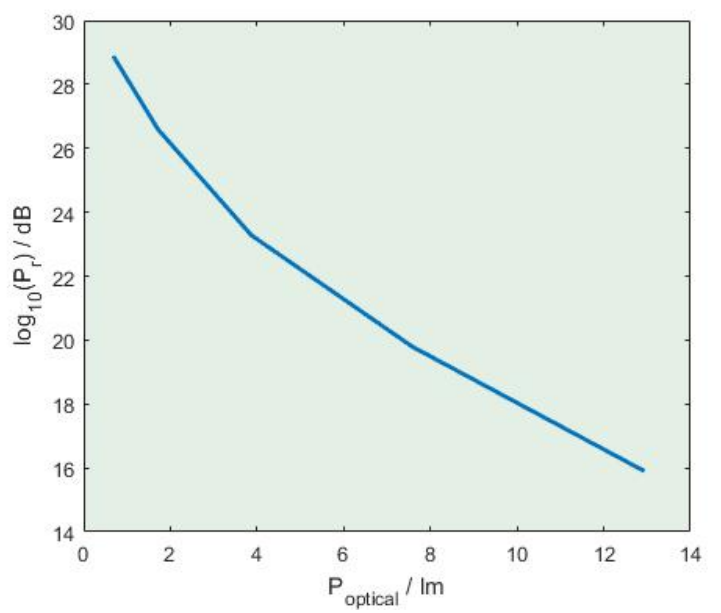

Fig. 3: Illumination Intensity vs Received Signal Power

What the result shown is counter common sense that the received power decrease withing the illumination intensity increasing. By analysing the formulas we derived above, the increase of the illumination is stimulated by the higher quiescent junction current $I_{Q}$, which will bring about the small signal resistance of $\mathrm{p}$-n junction $r_{D}$ diminishing. If the rest conditions fixed, the received signal power will decrease by less resistance $r_{D}$.

\section{EXPERIMENT AND RESULT}

In this section, the experiment has been implemented and the results have been illustrated.

\section{A. Experiment Set up}

Fig.4. presents a diagram of the experimental VLC system set-up. At the analogue transmitter front-end, the input signal generated from the Network Analyzer (Agilent E5061B) or function generator (Agilent E8257D) was first amplified and superimposed with the DC component by the driver circuit. The modulated light emitted from the InGaN based white LED was transmitted over an indoor VLC channel.

Light from the white LED was imaged onto a highspeed avalanche photodiode (AD500-8 TO). The APD has a detection wavelength range of $400-1100 \mathrm{~nm}$ with an active area of $0.2 \mathrm{~mm}^{2}$ and an $1 \mathrm{GHz}-3 \mathrm{~dB}$ bandwidth. The optical

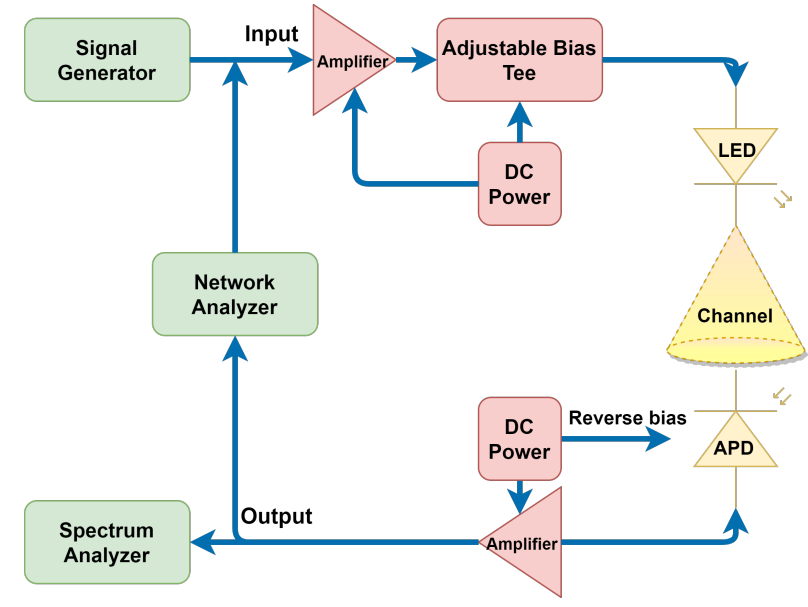

Fig. 4: Experiment Set up

signal from the LED was converted to electrical current signal through the APD and then the current signal was amplified to voltage signal by a low noise trans-impedance amplifier (TIA). The received electrical signal was collected by the Network Analyzer or the Spectrum analyzer(RS FS300).

\section{B. Effect of Illumination Intensity on VLC System Power Transfer function}

The power transfer function illustrates the energy efficient and transmission capability of the communication system. The experimental and theoretical relationship between illumination intensity and VLC system power transfer function from $5 \mathrm{MHz}$ to $15 \mathrm{MHz}$ are compared in Fig. 5 (a). The measurement and simulation at $10 \mathrm{~cm}$ distance are generated in difference illuminance: 400 lx, 1000 lx, 2000 lx, 4000 lx, 7000 lx which are lux-1 to lux-5 respectively. The predicted and measured power transfer function decrease with increasing the illumination intensity of LED and the comparison proved that the power transfer function model of VLC system link can predict successfully.

Based on the experiment and simulation results, the average power loss caused by the increased illuminance from lux-1 to lux-5 which are $1.9 \mathrm{~dB}, 4.7 \mathrm{~dB}, 7.5 \mathrm{~dB}, 11.1 \mathrm{~dB}$, in $10 \mathrm{~cm}$ distance respectively. By respecting the theory and experiment, the received signal power will extremely influenced by the illumination intensity of LED.

\section{Effect of Illumination Intensity on SNR of VLC system}

The predicted and measured effects of illumination intensity of LED on VLC system SNR performance is illustrated in Fig. 5 (b). According to the result, it is obvious that the communication quality will be greatly affected by the illumination intensity of LED. The SNR of VLC system will decrease approximately $3.6 \mathrm{~dB}$ per $1000 \mathrm{~lx}$ increase.

\section{CONCLUSION AND Discussion}

In this paper, from the perspective of the VLC system model, the effect of illumination intensity on a LED based VLC system is explained. In our experiment, we measured 


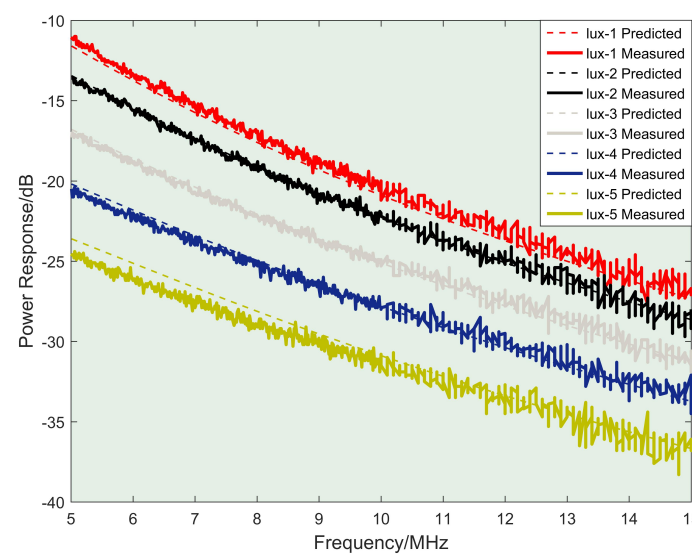

(a) The power response in different illuminance at $10 \mathrm{~cm}$

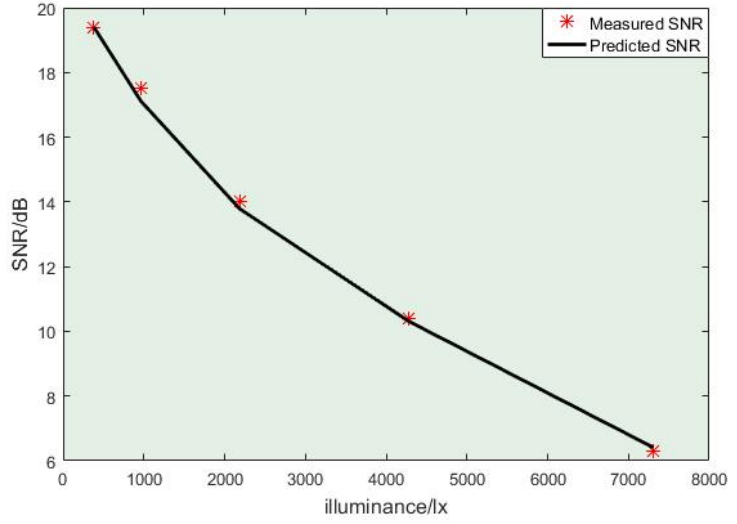

(b) The SNR in different illuminance at $10 \mathrm{~cm}$

Fig. 5: The Effect of Illumination intensity on VLC System Performance

the power transfer function and SNR by using a commercial white LED through a typical VLC system link. According to the experimental results, the SNR decreased approximately $3.6 \mathrm{~dB}$ with the illuminance rising per thousand lux, which well matched the prediction of the proposal system model. Moreover, the average power loss of the system increased from $1.9 \mathrm{~dB}$ to $11.1 \mathrm{~dB}$ under the illuminance from $400 \mathrm{~lx}$ to 7000 lx respectively.

According to the the European Lighting Standard EN124641 [14], the minimum illuminance of an indoor working area should be higher than 500 lx. In this background, our system model enable to evaluate the communication quality correctly on white LED based VLC system under specific illuminance requirements. However, there existed some challenges need to be covered. For instance, our proposal is under the assumption that the light propagation is the Lambertian directional. Besides, the influence of the different modulation schemes is not considering.

In the near future, we will further improve our system model through different modulation schemes. The objective is to realize a joint illumination and communication system which is not only on the advancement in the data rate but also to comply with common illumination requirements. It can guarantee that VLC-enabled luminaries can be used directly without any extra cost in the lighting market.

\section{ACKNOWLEDGMENT}

The authors gratefully acknowledge the financial support of the National Key R\&D Program of China (Grant No.2017YFE011230) and the EU Horizon 2020 program towards the Internet of Radio-Light project H2020-ICT 761992.

\section{REFERENCES}

[1] Chowdhury M Z, Hossan M T, Islam A, et al. A comparative survey of optical wireless technologies: Architectures and applications[J]. IEEE Access, 2018, 6: 9819-9840.

[2] Pirinen P. A brief overview of $5 \mathrm{G}$ research activities[C]//1st International Conference on 5G for Ubiquitous Connectivity. IEEE, 2014: 17-22.
[3] Jokanovic D, Josipovic M. RF spectrum congestion: resolving an interference case[C]//2011 IEEE International Conference on Microwaves, Communications, Antennas and Electronic Systems (COMCAS 2011). IEEE, 2011: 1-4.

[4] Tsiatmas A, Willems F M J, Linnartz J P M G, et al. Joint illumination and visible-Light Communication systems: Data rates and extra power consumption[C]//2015 IEEE International Conference on Communication Workshop (ICCW). IEEE, 2015: 1380-1386.

[5] Tsonev D, Videv S, Haas H. Towards a $100 \mathrm{~Gb} / \mathrm{s}$ visible light wireless access network[J]. Optics express, 2015, 23(2): 1627-1637.

[6] Shi, L., Li, W., Zhang, X., Zhang, Y., Chen, G., Vladimirescu, A. (2018, December). Experimental 5G New Radio integration with VLC. In 2018 25th IEEE International Conference on Electronics, Circuits and Systems (ICECS) (pp. 61-64). IEEE.

[7] Shi, L., Wang, W., Zhang, X., Vladimirescu, A., Wang, Z., Zhang, Y., Wang J. PAPR reduction based on deep autoencoder for DCO-OFDM VLC system. In 2019 IEEE International Symposium on Broadband Multimedia Systems and Broadcasting (BMSB). IEEE.

[8] L. Shi, X. Zhang, and A. Vladimirescu, "Experimental testbed for VLCbased localization framework in 5G Internet of Radio Light," in 2019 26th IEEE International Conference on Electronics, Circuits and Systems (ICECS), 2019

[9] "IEEE standard [ '1 and metropolitan networks-part 15.7:Short range wireless optical communication using visible light," lEEE Std 802.15.72011, . 1 -309, 2011.

[10] Wb Ding, F Yang, H Yang, Jt Wang, Xf Wang, X Zhang, j Song, ”A hybrid power line and visible light communication system for indoor hospital applications", Computer in Industry, vol.68. 2015.

[11] J Cosmas, Y Zhang, X Zhang, "Internet of radio-light: $5 \mathrm{~g}$ broadband in buildings", European Wireless, 2017.

[12] Y Tanaka, S. and . Nakagawa, "Wireless optical transmission with the white colored LEO [ the wireless home links," Proc. / the 11th lnt. Symp. Persona/, Indoor and / Radio Communications (PIMRC 2000), London,UK,. 1325-1329,2000.

[13] M Ishida, "InGaN based LEOs and their application," OPTRONICS, vol.19,.228,. 120-125,2000.

[14] Boyce P R. Human factors in lighting[M]. Crc Press, 2014.

[15] Gancarz J, Elgala H, Little T D C. Impact of lighting requirements on VLC systems[J]. IEEE Communications Magazine, 2013, 51(12): 34-41.

[16] Popadic M, Lorito G, Nanver L K. Analytical Model of I - V Characteristics of Arbitrarily Shallow pn Junctions[J]. IEEE Transactions on Electron Devices, 2008, 56(1): 116-125.

[17] Z. Ghassemlooy, W. Popoola, S. Rajbhandari "Optical Wireless Communications" March 31, 2017 Reference - 575 Pages - 268 B/W Illustrations ISBN 9781138074804 - CAT K34043 